Discrete Comput Geom 31:287-303 (2004)

DOI: $10.1007 / \mathrm{s} 00454-003-2902-0$

\title{
The Number of Embeddings of Minimally Rigid Graphs*
}

\author{
Ciprian Borcea ${ }^{1}$ and Ileana Streinu ${ }^{2}$ \\ ${ }^{1}$ Department of Mathematics, Rider University, \\ Lawrenceville, NJ 08648, USA \\ borcea@ rider.edu \\ ${ }^{2}$ Department of Computer Science, Smith College, \\ Northampton, MA 01063, USA \\ streinu@cs.smith.edu
}

\begin{abstract}
Rigid frameworks in some Euclidean space are embedded graphs having a unique local realization (up to Euclidean motions) for the given edge lengths, although globally they may have several. We study the number of distinct planar embeddings of minimally rigid graphs with $n$ vertices. We show that, modulo planar rigid motions, this number is at most $\left(\begin{array}{c}2 n-4 \\ n-2\end{array}\right) \approx 4^{n}$. We also exhibit several families which realize lower bounds of the order of $2^{n}, 2.21^{n}$ and $2.28^{n}$.

For the upper bound we use techniques from complex algebraic geometry, based on the (projective) Cayley-Menger variety $C M^{2, n}(C) \subset P_{\left(\begin{array}{c}n \\ 2\end{array}\right)-1}(C)$ over the complex numbers $C$. In this context, point configurations are represented by coordinates given by squared distances between all pairs of points. Sectioning the variety with $2 n-4$ hyperplanes yields at most $\operatorname{deg}\left(C M^{2, n}\right)$ zero-dimensional components, and one finds this degree to be $D^{2, n}=$ $\frac{1}{2}\left(\begin{array}{c}2 n-4 \\ n-2\end{array}\right)$. The lower bounds are related to inductive constructions of minimally rigid graphs via Henneberg sequences.

The same approach works in higher dimensions. In particular, we show that it leads to an upper bound of $2 D^{3, n}=\left(2^{n-3} /(n-2)\right)\left(\begin{array}{c}2 n-6 \\ n-3\end{array}\right)$ for the number of spatial embeddings with generic edge lengths of the 1-skeleton of a simplicial polyhedron, up to rigid motions.

Our technique can also be adapted to the non-Euclidean case.
\end{abstract}

\section{Introduction}

In this paper we are concerned with graph embeddings subject to edge length constraints. We use embedding (or realization) in the extended sense, which allows some vertices to

* Ileana Streinu was supported by NSF Grants CCR-0105507 and CCR-0138374. 
coincide and some edges to cross. For a given graph and for a given set of edge lengths, a natural question to ask is: how many embeddings in $R^{d}$ are there?

Obviously, for a fixed dimension $d$, some graphs have a continuum of embeddings, or may have no embedding at all for particular choices of edge lengths.

We consider minimally rigid graphs on $n \geq d+1$ vertices in dimension $d$ (which have $d n-\left(\begin{array}{c}d+1 \\ 2\end{array}\right)$ edges and unique local realizations for generic choices of edge lengths), with particular regard to dimensions 2 and 3.

Our results give a general upper bound in arbitrary dimension, which is of the order of $2^{d n}$ for fixed $d$ and $n$ sufficiently large. We also exhibit a family of graphs inducing a lower bound of the order of $2.28^{n}$ in dimension 2 .

Using the same technique we obtain upper bounds in the non-Euclidian case (spherical or hyperbolic).

Historical Perspective. Distance geometry relies on foundational work of Cayley on configurations of $n$ points in Euclidean $d$-space. As elaborated by Menger (see p. 237 of [4]), this led to conditions characterizing systems of $\left(\begin{array}{l}n \\ 2\end{array}\right)$ positive reals that arise as pairwise squared distances between $n$ points in $R^{d}$. See also [5] and [13]. In dimension 3, distance geometry has been used for the study of molecular conformation in chemistry [10], [11], [20]. Indeed, inter-atomic distance information can be obtained from nuclear magnetic resonance spectra of a molecule. Solving the graph embedding problem determines coordinates for the atoms, and hence the three-dimensional shape of the molecule. Other applications include surveying and satellite ranging.

Our investigation is also related to classical studies in the kinematics of mechanical linkages, in particular the problem of tracing algebraic curves. Wunderlich [37] gives an interesting family of generalized planar coupler curves with degree growing exponentially in the number of links. Related work was also done in combinatorial rigidity theory [9], [35], [17]. Rigid frameworks are embedded graphs having a unique local realization for the given edge lengths, up to rigid motions. However, globally there may be several realizations. Using special combinations of graphs and edge lengths, Saxe [30] has shown that it is NP-hard to solve the graph embedding problem in dimension 2, as well as to determine whether it has a unique solution (up to reflections). Under the assumption of genericity, Hendrickson [21] studied conditions on rigid frameworks that guarantee a unique global realization. Recently, Berg and Jordán [3] gave an elegant characterization of graphs with a minimum number of edges and generic unique realizations ("rigidity circuits".)

Minimally Rigid Graphs. Hendrickson's graphs, as well as the complete graphs implicit in the Cayley-Menger conditions, are redundant in terms of distance dependencies. The problem of reconstructing point sets from distances is more challenging for them, in the sense that small perturbation errors in the input data will render the problem infeasible. In this paper we go to the lower end of the dependency spectrum and study the number of embeddings of minimally rigid graphs in dimension d (see Definition 2.1.)

In dimension 2 these graphs possess a simple combinatorial characterization [25], and, for generic edge lengths, will have a discrete realization space. We refer to these graphs as Laman graphs. In dimension 3 one does not have a comparable result. However, as observed by Gluck [16], a combination of arguments going back to Cauchy, Steinitz, 
Dehn, Weyl and Alexandrov ensures that 1-skeleta of simplicial polyhedra are minimally rigid graphs in dimension 3 .

Techniques and Results. Our approach emphasizes the possibility of exploiting the algebraic character of the prevalent relations in distance geometry by rephrasing the problems in terms of associated complex projective varieties.

Indeed, our upper bounds are derived from degree computations for the complex projective varieties $C M^{d, n}$ induced by the Cayley-Menger determinant equalities for squared distances of $n$ points in $R^{d}$. In [6] these Cayley-Menger varieties are identified with classical determinantal varieties of symmetric forms.

For planar ${ }^{1}$ realizations of Laman graphs we obtain:

Theorem 1.1. Given a generic choice of edge lengths, a Laman graph with $n$ vertices has at most $\left(\begin{array}{c}2 n-4 \\ n-2\end{array}\right)$ planar embeddings, up to rigid motions.

In the direction of lower bounds, we exhibit several families which realize bounds of the order of $2^{n}, 2.21^{n}$ and $2.28^{n}$.

For spatial $(d=3)$ realizations of 1-skeleta of simplicial polyhedra we obtain:

Theorem 1.2. Given a generic choice of edge lengths, the 1-skeleton of a simplicial complex which has $n$ vertices and is topologically a two-dimensional sphere has at most $\left(2^{n-3} /(n-2)\right)\left(\begin{array}{c}2 n-6 \\ n-3\end{array}\right)$ spatial embeddings, up to rigid motions.

In general, we have:

Theorem 1.3. Let $d$ be a given dimension. Let $\mathcal{G}$ be a class of connected graphs with $n \geq d+1$ vertices and $m=d n-\left(\begin{array}{c}d+1 \\ 2\end{array}\right)$ edges such that each graph allows an infinitesimally rigid realization in $R^{d}$. Then, for a generic choice of edge lengths, each graph in $\mathcal{G}$ has a finite number of embeddings in $R^{d}$ and this number is bounded from above by $2 D^{d, n}$. Here $D^{d, n}$ stands for the degree of the Cayley-Menger variety $C M^{d, n}(C) \subset P_{\left(\begin{array}{c}n \\ 2\end{array}\right)-1}(C)$ and is given by the formula

$$
D^{d, n}=\prod_{k=0}^{n-d-2} \frac{\left(\begin{array}{c}
n-1+k \\
n-d-1-k
\end{array}\right)}{\left(\begin{array}{c}
2 k+1 \\
k
\end{array}\right)}
$$

For $d$ constant and $n$ sufficiently large, $D^{d, n} \approx 2^{d n}$.

It is worth noticing that general techniques based on real algebraic geometry (OleinikPetrovskii-Milnor-Thom bounds) yield less good upper bounds (see Section 2).

\footnotetext{
${ }^{1}$ Throughout the paper the term planar is used in its geometric sense to refer to objects in dimension $d=2$. The reader should not interpret it to mean non-crossing as in the graph drawing literature.
} 
The Euclidean results can be adapted to the non-Euclidean setting to yield:

Theorem 1.4. Let $d$ be given and assume a generic choice of edge lengths (subject to $0<l_{i j}<\pi$ in the spherical case). A $d$-minimally rigid graph with $n \geq d+1$ vertices has at most

$$
2 D^{d+1, n+1}=2 \prod_{k=0}^{n-d-2} \frac{\left(\begin{array}{c}
n+k \\
n-d-1-k
\end{array}\right)}{\left(\begin{array}{c}
2 k+1 \\
k
\end{array}\right)}
$$

realizations in the sphere $S^{d}$, or the hyperbolic space $H^{d}$, up to orientation preserving isometries (i.e. rigid motions).

Future Perspectives and Open Questions. Trying to bridge the still significant gap between the upper and lower bounds can be approached from both directions. The upper bound is presumably high, as it counts not only "realistic" solutions, but also real solutions which do not represent configurations and complex solutions in the CayleyMenger variety.

On the other hand, in showing that Laman graphs which require Henneberg II constructions may have more than $c^{n}, c>2$, planar embeddings, we give evidence that the lower bound may possibly be raised.

An important problem for $d \geq 3$ is to identify and characterize other classes of minimally rigid graphs, besides 1-skeleta of triangulated spheres and 3-simple graphs. Such examples could potentially produce improved lower bounds in dimension 3 .

\section{Definitions and Preliminaries}

In this section we define the necessary concepts: configuration space, rigid, infinitesimally rigid, $d$-minimally rigid graph. We also formulate the problem and, for the sake of comparison, use standard results from real algebraic geometry to derive upper bounds that we later improve.

Rigidity Theoretic Definitions. Let $G$ be a graph $G=(V, E), V=\{1, \ldots, n\}$, $m=|E|$. A framework $(G, L)$ is a graph $G$ together with a set $L=\left\{l_{i j} \mid i j \in E\right\}$ of positive numbers $l_{i j}>0$ interpreted as edge lengths associated to the edges. A realization or embedding $G(P)$ of $(G, L)$ in some space $R^{d}$ is given by a mapping of the vertices onto a set of points $P=\left\{p_{1}, \ldots, p_{n}\right\} \subset R^{d}$ such that $l_{i j}$ equals the Euclidian distance between the two points $p_{i}$ and $p_{j}$. Note that edges may cross and vertices may coincide.

Notation. The squared distance (resp. the distance) between two points labeled $i$ and $j$ will be denoted by $d_{i j}$ (resp. $l_{i j}$ ) when given a priori, and by $x_{i j}$ (squared distance only) when unknown.

The realization or configuration space of $(G, L)$ in $R^{d}$ is the set of all possible realizations, modulo the $\left(\begin{array}{c}d+1 \\ 2\end{array}\right)$-dimensional group of rigid motions. Obviously if a graph has one embedding, then its mirror image is also an embedding. In this paper we consider as distinct two embeddings differing by a reflection: this is a simple technicality that does not affect the asymptotic bounds we derive.

Any graph has some embedding, for some edge lengths, but for given values of $L$ 
the configuration space may be empty, finite or higher-dimensional. A realization $G(P)$ is rigid or locally unique when it cannot be deformed continuously into another (noncongruent) realization of the same framework (see [9] or [35] for precise definitions.) Otherwise, the realization is just one point of a higher-dimensional component of the realization space, and the embedded graph in this case is called a mechanism, as it is flexible. The dimension of the local component to which the realization belongs is its number of degrees of freedom. A one-degree-of-freedom mechanism is an embedded framework whose component is one-dimensional (i.e. a curve).

The rigidity matrix $A$ associated to a realization $G(P)$ is the following $m \times d n$ matrix. The rows are indexed by the $m$ edges $i j \in E$ and the $d n$ columns are grouped into $d$-tuples corresponding to the $d$ coordinates of a point indexed by $i, i=1, \ldots, n$. The $i j$ th row has 0 entries everywhere, except in the $i$ th and $j$ th $d$-tuples of columns, where the entries are $p_{i}-p_{j}$, resp. $p_{j}-p_{i}$ :

$$
i j\left(\begin{array}{ccccccc}
1 & \cdots & i & \cdots & j & \cdots & n \\
0 & \cdots & & & & & \\
\cdots & & p_{i}-p_{j} & \cdots & p_{j}-p_{i} & \cdots & 0
\end{array}\right) .
$$

A solution $v \in R^{d n}$ of the linear system $A v=0$ is called an infinitesimal motion. It is trivial if it arises from an infinitesimal rigid transformation of $R^{d}$. The space of trivial infinitesimal motions is a $\left(\begin{array}{c}d+1 \\ 2\end{array}\right)$-dimensional linear subspace of the space of solutions of $A v=0$. Hence the rank of the rigidity matrix is at most $d n-\left(\begin{array}{c}d+1 \\ 2\end{array}\right)$. It is known that it depends on both the combinatorial structure of the graph $G$ and on the points of the embedding $P$.

A realization is infinitesimally rigid if the rank of its associated rigidity matrix is precisely $d n-\left(\begin{array}{c}d+1 \\ 2\end{array}\right)$. This means that the only solutions $v \in R^{d n}$ of the system $A v=0$ are the trivial infinitesimal motions. An infinitesimally rigid configuration is rigid, but the opposite is not true: there exist rigid embeddings which are not infinitesimally rigid.

Definition 2.1. For a given dimension $d$, we say that a graph with $n \geq d+1$ vertices is $d$-minimally rigid or minimally rigid in dimension $d$ if it has $m=n d-\left(\begin{array}{c}d+1 \\ 2\end{array}\right)$ edges and allows an infinitesimally rigid realization in $R^{d}$. These graphs are also called $d$-isostatic (see [17].)

Remark. As a maximal rank condition, infinitesimal rigidity will hold true on the complement of an algebraic subvariety if holding true at one point. Consequently, a $d$ minimally rigid graph, as defined above, will have only infinitesimally rigid realizations in $R^{d}$, provided $d_{i j}=l_{i j}^{2}, i j \in E$, are chosen in the complement of a certain algebraic subvariety (i.e. generically as the term is used in algebraic geometry).

\section{Dimension Two}

Laman [25] gave a complete characterization of 2-minimally rigid graphs: we call them Laman graphs. They have $2 n-3$ edges, and each subset of $k$ vertices spans at most $2 k-3$ edges. 


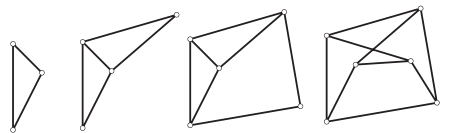

(a)

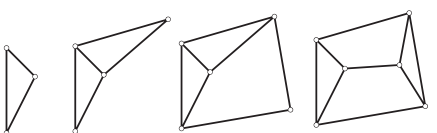

(b)

Fig. 1. Henneberg constructions for two graphs on six vertices, using type I and type II steps. (a) $K_{3,3}$, (b) the Desargues graph.

Henneberg Sequences. Laman graphs have many elegant combinatorial properties. The so-called Henneberg sequences (defined after [22]) are used to construct them in an inductive fashion (see [32]). A Henneberg sequence for a graph $G$ is a sequence $G_{3}, G_{4}, \ldots, G_{n}$ of Laman graphs on $3,4, \ldots, n$ vertices, such that: $G_{3}$ is a triangle, $G_{n}=G$ and each graph $G_{i+1}$ is obtained from the previous one, $G_{i}$, via one of two types of steps: type I and type II. A Henneberg step of type I adds a new vertex and two new edges connecting this vertex to two arbitrary vertices of $G_{i}$. A Henneberg step of type II adds a new vertex and three new edges, and removes an old edge, more precisely: the three new edges must connect the new vertex to three old vertices such that at least two of them are joined via an edge; that edge will be removed. See Fig. 1.

Not all Laman graphs can be constructed using only type I steps. We call those which can Henneberg I graphs ${ }^{2}$ and those which cannot Henneberg II. In particular, triangulations of planar polygons are Henneberg I graphs. The smallest examples of Henneberg II graphs are shown in Fig. 1. The one on the right, the so-called Desargues framework, borrows its name from the classical Desargues configuration (two triangles in perspective) from plane projective geometry, which it induces for some special edge lengths. As an aside, we note that these special embeddings, illustrated in the last two cases of Fig. 2, are not infinitesimally rigid, or may even be flexible. Such embeddings are not generic, and therefore not treated in the general discussion of this paper. The first case in Fig. 2 is an example of a generic embedding.

\section{Dimension Three}

A combinatorial characterization of minimally rigid graphs in dimension 3 or higher is not yet known. However, there is an important class of graphs which are minimally rigid
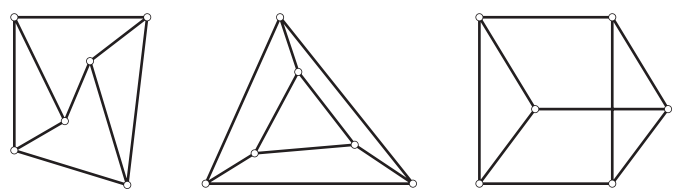

Fig. 2. The Desargues framework and three types of its possible embeddings: infinitesimally rigid, rigid but not infinitesimally rigid and flexible.

\footnotetext{
${ }^{2}$ These graphs are called 2-simple in the engineering literature. See [34] and the references given there.
} 
in dimension 3, namely the 1-skeleta of simplicial convex polyhedra. By a theorem of Steinitz [31], these are the maximal (with respect to the set of edges) 3-connected graphs which admit planar non-crossing realizations (i.e. the maximally planar graphs or planar triangulations, as they are known in the graph drawing literature). Any realization of such a graph in dimension 3 as a 1-skeleton of a simplicial convex polyhedron is infinitesimally rigid (see [8], [12] and [16]).

\section{Higher Dimensions}

Here is a simple argument showing that there exist generic minimally rigid graphs in any dimension $d$. Consider the class of graphs constructed inductively as follows. Start with the 1-skeleton of a $d$-simplex (the complete graph on $d$ vertices). At each step afterwards, add a new vertex and $d$ edges connecting it to $d$ of the previously constructed vertices. It is straightforward to construct an infinitesimally rigid embedding of this graph, by embedding the vertices on a set of points in general position (an induction on the construction shows that the rank of the rigidity matrix is as expected). Note that this is the natural generalization of the Henneberg I construction from dimension 2. Such graphs are called $d$-simple in the engineering literature (see [34].) It would be interesting to find other classes of minimally rigid graphs with comparable simple descriptions.

The Embedding Problem. Given a framework $(G, L)$, one is interested in understanding its configuration space; in particular, topological invariants such as the number of connected components and their dimensions.

The configuration space is the orbit space of the group of rigid motions acting on the affine algebraic variety defined in $R^{d n}$ by the equations

$$
\left|p_{i}-p_{j}\right|^{2}=d_{i j}, \quad i j \in E
$$

Since the group is connected, the number of connected components can be counted in the algebraic variety itself.

Bounds from Real Algebraic Geometry. Consider an algebraic system of $s$ equations of degree at most $D$ in $k$ variables $P_{l}\left[x_{1}, \ldots, x_{k}\right]=0, l=1, \ldots, s$, degree $P_{l} \leq D$. Its real solutions form a real algebraic set.

The following result from real algebraic geometry bounds the number of connected components or, more generally, the Betti numbers of an algebraic set.

Oleinik-Petrovskii-Milnor-Thom Theorem ([28], [26], [33], see also [2]). For equations of degree at most $D$, in $k$ variables, the sum of the Betti numbers (and hence the number of connected components) is at most $D(2 D-1)^{k-1}$.

Applying the theorem to a minimally rigid graph in dimension $d$ : the system has $m=d n-\left(\begin{array}{c}d+1 \\ 2\end{array}\right)$ equations of degree 2 in $d n$ variables. The Oleinik-Petrovsky-MilnorThom bound implies at most $2 \times 3^{d n-1} \approx\left(3^{d}\right)^{n}$ components (i.e. realizations). In particular, in dimension 2 the bound is of the order $9^{n}$. 
A better bound can be obtained as follows: first use rigid motions to reduce the number of variables to the number of equations and then apply the refined Bézout Theorem [14] to the complex projective extension of the space of real variables. More precisely, translations eliminate $d$ variables by fixing $p_{1}=0$, then rotations can bring $p_{2}$ along the first axis, then rotations fixing this axis can bring $p_{3}$ in the plane of the first two axes, and so on, ending with $p_{d}$ in the subspace $x_{d}=0$. This reduces the space of variables from $R^{\text {nd }}$ to

$$
R^{n d-\left(\begin{array}{c}
d+1 \\
2
\end{array}\right)} \subset P_{n d-\left(\begin{array}{c}
d+1 \\
2
\end{array}\right)}(C),
$$

where the length conditions define precisely $|E|=n d-\left(\begin{array}{c}d+1 \\ 2\end{array}\right)$ quadratic equations. The refined Bézout Theorem then gives the upper bound $2^{n d-\left(\begin{array}{c}d+1 \\ 2\end{array}\right)}$ for the number of isolated solutions.

We will obtain an even better upper bound for the number of realizations by using a different algebraic object, the Cayley-Menger variety.

\section{Cayley-Menger Varieties}

A set of $\left(\begin{array}{l}n \\ 2\end{array}\right)$ positive numbers $d_{i j}=l_{i j}^{2}, i, j=1, \cdots, n, i<j$, has to satisfy certain algebraic conditions to be the set of squared Euclidian distances between $n$ points in $R^{d}$. Some of these conditions are equalities (due to Cayley) and express the fact that the rank of the following matrix is at most $d+2$ :

$$
\left(\begin{array}{lllll}
0 & 1 & 1 & \cdots & 1 \\
1 & 0 & d_{12} & \cdots & d_{1 n} \\
1 & & \cdots & & \\
1 & d_{n 1} & d_{n 2} & \cdots & 0
\end{array}\right)
$$

The other conditions, due to Menger, are inequalities.

If we restrict our attention only to the equalities, we are led to consider a complex projective variety $C M^{d, n}(C) \subset P_{\left(\begin{array}{l}n \\ 2\end{array}\right)-1}(C)$, which is called a Cayley-Menger variety in [6]. Here $C$ stands for the field of complex numbers, to emphasize that we refer to the complex and not the real variety. It is defined by setting to zero all the $(d+3) \times(d+3)$ minors of the following symmetric matrix:

$$
\left(\begin{array}{lllll}
0 & 1 & 1 & \cdots & 1 \\
1 & 0 & x_{12} & \cdots & x_{1 n} \\
1 & & \cdots & & \\
1 & x_{n 1} & x_{n 2} & \cdots & 0
\end{array}\right),
$$

where $\left(x_{i j}\right)_{1 \leq i<j \leq n}$ are complex homogeneous coordinates in the projective space $P_{\left(\begin{array}{c}n \\ 2\end{array}\right)-1}(C)$.

We arrive in this manner to a complex-projective formulation of our Embedding Problem: Solve the system consisting of the Cayley determinantal equations, plus the linear conditions setting the graph edges to prescribed lengths. The latter conditions have the form

$$
\frac{x_{i j}}{x_{k l}}=\frac{d_{i j}}{d_{k l}}, \quad \text { that is, } \quad d_{k l} x_{i j}=d_{i j} x_{k l} \quad \text { for } \quad i j, k l \in E,
$$


which (for $m=|E|=d n-\left(\begin{array}{c}d+1 \\ 2\end{array}\right)$ edges) amounts to $d n-\left(\begin{array}{c}d+1 \\ 2\end{array}\right)-1$ independent hyperplane sections.

As we are about to see, this is precisely the (complex) dimension of the CayleyMenger variety $C M^{d, n}(C)$. Hence, by a general result in complex projective geometry, we have:

Proposition 3.1. A codimension $d n-\left(\begin{array}{c}d+1 \\ 2\end{array}\right)-1$ linear section of the Cayley-Menger variety $C M^{d, n}(C)$ has at most $\operatorname{deg}\left(C M^{d, n}(C)\right)$ isolated points.

Here, $\operatorname{deg}\left(C M^{d, n}(C)\right)$ stands for the degree of our variety, which is defined as the number of (isolated) points in a generic codimension $d n-\left(\begin{array}{c}d+1 \\ 2\end{array}\right)-1$ linear section.

Remark. Even with generic choices of edge lengths $\left(l_{i j}\right)_{i j \in E}$, the linear sections corresponding to $d$-minimally rigid graphs remain very peculiar, and are never generic in the sense required in the definition of the degree. However, we know that a generic choice of edge lengths ensures that all configuration solutions (which we also call "realistic", meaning both real and satisfying Menger's inequalities) are isolated points, because the resulting framework is infinitesimally rigid.

Corollary 3.2. For a generic choice of edge lengths, a d-minimally rigid graph with $n$ vertices has at most $2 \operatorname{deg}\left(C M^{d, n}(C)\right)$ distinct planar embeddings, up to rigid motions.

Indeed, there will be twice as many solutions to the original embedding problem than "realistic" solutions in the sectioning of the Cayley-Menger variety, because we count as distinct two realizations one of which is the reflection of the other (modulo rigid motions in the plane), while the Cayley-Menger approach automatically identifies congruent configurations.

\section{The Upper Bounds in the Euclidean case}

We begin with a "naive" count for dimension: $n$ points in $R^{d}$ require $d n$ parameters; equivalence under Euclidean motions and rescaling cuts down $\left(\begin{array}{c}d+1 \\ 2\end{array}\right)+1$ parameters, so that the configuration space for $n$ (ordered) points in the plane, modulo congruence and similarity, should be $\left(d n-\left(\begin{array}{c}d+1 \\ 2\end{array}\right)-1\right)$-dimensional. The complex version $C M^{d, n}(C)$ (which is, technically, the Zariski-closure of the configuration space in $P_{\left(\begin{array}{l}n \\ 2\end{array}\right)-1}(C)$ ) should have as many complex dimensions.

In order to be precise, we first relate Cayley coordinates and Gram coordinates for a configuration of $n$ points $p_{1}, \ldots, p_{n} \in R^{d}$.

The Cayley coordinates are

$$
x_{i j}=x_{i j}(p)=\left|p_{i}-p_{j}\right|^{2}=\left\langle p_{i}-p_{j}, p_{i}-p_{j}\right\rangle, \quad 1 \leq i, j \leq n,
$$

with $\langle$,$\rangle denoting the usual inner product in R^{d}$. 
For Gram coordinates we have to choose one point as origin, and we make the choice $p_{1}=0$. Then we set

$$
y_{i j}=y_{i j}(p)=\left\langle p_{i}-p_{1}, p_{j}-p_{1}\right\rangle=\left\langle p_{i}, p_{j}\right\rangle, \quad 2 \leq i, j \leq n .
$$

The relation is simply the cosine theorem:

$$
y_{i j}=\frac{1}{2}\left(x_{1 i}+x_{1 j}-x_{i j}\right), \quad 2 \leq i, j \leq n .
$$

Normally, one looks at the Gram coordinates as arranged in a symmetric $(n-1) \times(n-1)$ matrix $Y$ with entries $y_{i j}=y_{j i}$, while we have seen above the Cayley coordinates arranged in a bordered symmetric $(n+1) \times(n+1)$ matrix, which we denote by $X$.

It is a simple exercise (see [4] or [6]) to observe the rank relation

$$
\operatorname{rk}(X)=2+\operatorname{rk}(Y)
$$

Since for configurations in $R^{d}$ the Gram matrix obviously has rank at most $d$, we see the reason for defining the Cayler-Menger variety by the vanishing of all $(d+3) \times(d+3)$ minors in $X$. In fact, we have:

Proposition 4.1. The passage from Cayley coordinates to Gram coordinates identifies the Cayley-Menger variety $C M^{d, n}(C)$ with the determinantal variety defined (projectively) by all complex (non-trivial) symmetric $(n-1) \times(n-1)$ matrices of rank at most d.

This brings the matter into the territory of classical algebraic geometry, and we may simply refer to [18] and [14]. The degree computation goes back to Giambelli (see [15], [19, Proposition 12(b), p. 78] and [24]).

The result converts our Corollary 3.2 into:

Theorem 4.2. Let $d$ be given and assume a generic choice of edge lengths. A $d$ minimally rigid graph with $n$ vertices has at most

$$
2 D^{d, n}=2 \prod_{k=0}^{n-d-2} \frac{\left(\begin{array}{c}
n-1+k \\
n-d-1-k
\end{array}\right)}{\left(\begin{array}{c}
2 k+1 \\
k
\end{array}\right)}
$$

embeddings in $R^{d}$, up to rigid motions.

This is our Theorem 1.3 in the Introduction. A direct calculation shows

$$
\frac{D^{d, n+1}}{D^{d, n}}=\prod_{k=1}^{d} \frac{2 n-d-k}{n-k}<2^{d},
$$

hence

$$
2 D^{d, n}<2^{d n-2\left(\begin{array}{c}
d+1 \\
2
\end{array}\right)+1} .
$$

The stated bound is clearly less than the bound of $2^{d n-\left(\begin{array}{c}d+1 \\ 2\end{array}\right)}$ obtained via the refined Bézout Theorem. 
For dimension 2 we have:

Theorem 4.3. Given a generic choice of edge lengths, a Laman graph with $n$ vertices has at most $2 \operatorname{deg}\left(C M^{2, n}(C)\right)=\left(\begin{array}{c}2 n-4 \\ n-2\end{array}\right)$ planar embeddings, up to rigid motions.

A direct computation of this particular degree is given in [6].

In dimension 3 we obtain:

Theorem 4.4. Given a generic choice of edge lengths, the 1-skeleton of a simplicial convex polyhedron with $n$ vertices has at most $2 \operatorname{deg}\left(C M^{3, n}(C)\right)=\left(2^{n-3} /(n-2)\right)\left(\begin{array}{c}2 n-6 \\ n-3\end{array}\right)$ embeddings in $R^{3}$, up to rigid motions.

\section{Lower Bounds}

The simplest family of examples yielding an exponential lower bound is given by triangulations of simple polygons. Indeed, any triangle may be flipped over an adjacent internal diagonal, giving a total of exactly $2^{n-3}$ embeddings. Taking into account the mirror image of the whole graph doubles the number of possibilities to $2^{n-2}$. See Fig. 3 .

Our goal in this section is to improve this trivial bound. We do so by refining the analysis of minimally rigid graphs via Henneberg constructions. We show first that Henneberg I graphs have at most as many embeddings as triangulations have, and for particular choices of edge lengths this number is achieved. Then we improve the lower bound twice, using two types of special iterations of Desargues graphs.

Bounds on the Number of Embeddings for Henneberg I Graphs. We first analyze the Henneberg constructions for triangulations of polygons. Starting from any triangle, at each step we add a new vertex and two new edges connecting it to two old vertices which are already joined by an edge, hence at a fixed distance. When the edge lengths are given, this construction is easily carried out using ruler and compass. The newly added vertex can be chosen to be one of the two intersections of two circles with radii the edge lengths of the newly added edges (which are known to intersect, as the edge
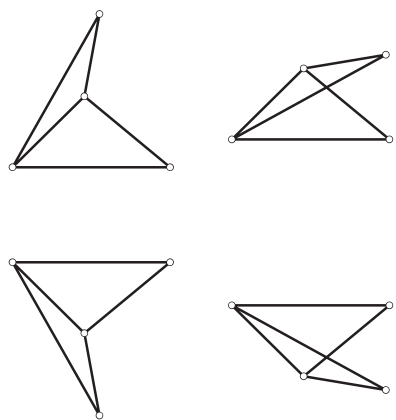

Fig. 3. Triangulations of polygons have $2^{n-2}$ embeddings. An example for $n=4$. 
lengths are computed from an existing triangulation). Any choice at step $i$ is valid and does not affect the number of further options.

We now see that this analysis depends only on the fact that, at step $i$ in the Henneberg I construction, the two vertices to be joined by the newly added edges to the new vertex are themselves connected by an edge, hence at a fixed distance. This amounts to "adding a triangle" to the previously constructed graph. Therefore, for a realizable choice of edge lengths (which always exists) we get:

Lemma 5.1. Any realizable Henneberg I graph obtained by "adding triangles" has exactly $2^{n-2}$ embeddings.

In the general Henneberg I step, the two added edges may not form a triangle with an old edge. Therefore the distances between relevant points (such as those used to add two new diagonals at the next step) may vary between embeddings constructed up to step $i$, and this may render the next step infeasible (i.e. having two complex instead of two real solutions). However, we can carry out the Henneberg I construction and choose good edge lengths at each step, which will guarantee that all subsequent steps yield real solutions. To do so, we simply choose the edge lengths of the two new edges to be approximately equal, and sum up to a bit over the maximum distance (over all embeddings of the Henneberg I graph $G$ to which this step is being applied) between the endpoints in $G$ of the new edges. Hence:

Proposition 5.2. Any realizable Henneberg I graph has at most $2^{n-2}$ embeddings. For any Henneberg I graph, there exists a choice of edge lengths such that the resulting framework has exactly $2^{n-2}$ embeddings.

Number of Embeddings for the Desargues Framework. To beat this bound we must use Henneberg II graphs. For special edge lengths, the Desargues graph gives the smallest example with more than $2^{n-2}$ embeddings. Namely, on six vertices, it can have up to 24 $\left(>2^{6-2}=16\right)$ embeddings.

Lemma 5.3. There exist edge lengths for the Desargues graph which induce 24 embeddings.

First, notice that if you remove an edge from a Laman graph, the resulting graph is a one-degree-of-freedom mechanism. Once an edge is pinned down, the other vertices trace algebraic curves. The curve of the largest degree could then be intersected with a circle whose center is placed at another vertex to get intersection points allowing us to place a new bar between the center of the circle and one of these intersection points. Notice also that such mechanisms contain several rigid subcomponents: the distances between vertices inside one such component stay fixed, no matter how the mechanism is moving.

Henneberg II graphs must contain at least one vertex of degree 3. If that vertex (and its adjacent edges) are removed, the resulting graph is a mechanism, whose vertices trace curves. The intersection of these curves with circles should (in general) be relatively easy to compute (even graphically, using for instance the Cinderella [29] software). To 

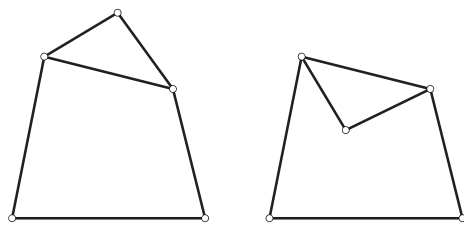

Fig. 4. The 4-bar mechanism obtained from the Desargues graph, in two embeddings of the coupler triangle.

be able to put the three edges easily back in a way that would give as many embeddings as possible for the given edge lengths, it would help if two of these edges would stay at a fixed distance (i.e. have an edge between them, or belong to a rigid component of the mechanism, which we will assume is grounded when the curve is traced). To achieve this goal, we use a floating center idea: place a circle anywhere, of any radius, as long as it gives the desired number of crossings with the curve traced by the mechanism. Then join the center via two bars to the grounded bar of the mechanism: this yields the grounded triangle of the Desargues configuration. To complete the construction, add a bar between the center of the circle and any of the intersection points. This can be done in as many ways as we had crossings. An additional set of embeddings is obtained by flipping (about the grounded edge) the two edges that were used to ground the center of the floating circle.

It turns out that the Desargues graph has all the properties to make this construction work.

The construction is illustrated in Figs. 4 and 5. Figure 4 shows, in two embeddings, the mechanism obtained by removing a vertex of degree 3 . It is assumed that the bottom edge is grounded and the motion of the mechanism is guided by the rotation of one of the adjacent edges around a grounded vertex. The curves traced by the degree 2 vertex of the

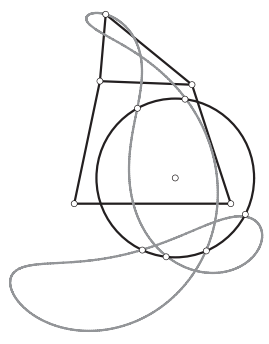

(a)

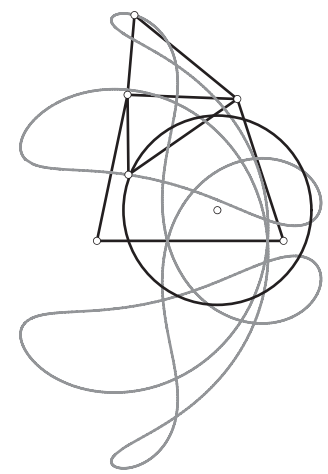

(b)

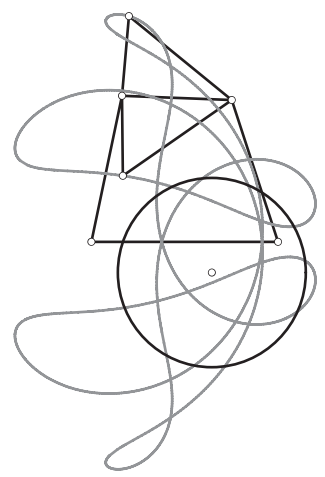

(c)

Fig. 5. (a) one of the coupler curves, the "floating" circle and their six crossings. The bottom bar is grounded to the plane, the upper three vertices are mobile and the top vertex traces the curve. (b) The two coupler curves and a placement of a circle crossing each of them in six points. (c) The symmetric placement of the circle from the middle picture induces another 12 crossings. 
triangle attached to this 4-bar mechanism have received a lot of attention in mechanical engineering (kinematics): indeed, in the 1950s a whole atlas of such "coupler curves" was published ([23], see also [27]). It is known that they are curves of degree 6 .

We have used Cinderella [29] to get a favorable arrangement for the coupler curves. Figure 5(a) shows one curve and a position of a "floating" circle intersecting it in six points. As a complex projective curve, the coupler curve has triple points at the two circular points at infinity [37]. Since any circle passes through these circular points, we always have six intersection points at infinity. By Bézout's theorem, a conic and a sextic meet in 12 points, hence in the real plane we can have at most six intersection points between a "floating"circle and the coupler curve. The static black-and-white pictures shown here do not capture the kinematic intricacy of the arrangement. A Cinderella applet allowing the user to vary the edge lengths and experiment with various shapes of the curves is available from http: / / cs.smith. edu/ streinu/Research/Embed/coupler.html.

The two flipped triangles on the coupler edge give two distinct and symmetric curves of degree 6 each. We can find the position of a circle intersecting each of them in six points, see Fig. 5(b). The symmetric arrangement gives another 12 crossings, for a total of 24. See Fig. 5(c). This proves Lemma 5.3.

Iterating the Desargues Framework. A simple observation for an iterative construction is that if we glue together, along any edge, two Desargues frameworks, we get a Laman graph. For instance, we could glue them in a "caterpillar" fashion, as in Fig. 6(a). The structure of this construction is illustrated in Fig. 6(b): it is a planar graph with quadrilateral faces (obtained from the Desargues graphs by retaining just one of the underlying 4-bar mechanisms used in obtaining many embeddings) and whose dual graph is a path. Any such gluing of quadrilaterals whose dual is a tree would give the same bound. Intuitively, this is the equivalent of gluing together triangles to get a polygon triangulation (the dual of a polygon triangulation being a tree).

Lemma 5.4. There exist edge lengths for which the number of embeddings of the iterated "caterpillar" Desargues frameworks is of the order of $24^{n / 4} \approx(2.21)^{n}$.

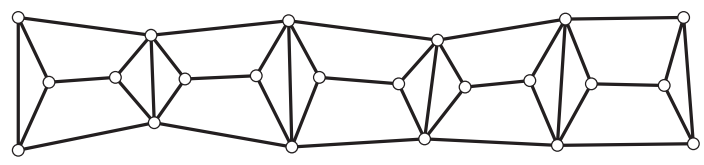

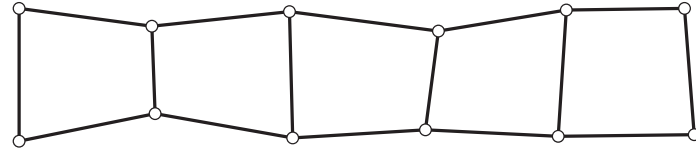

(a)

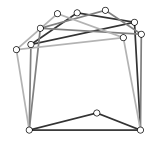

(b)

Fig. 6. (a) The "caterpillar" construction and its underlying structure. (b) The structure of the "fan" construction. 
Proof. Each Desargues graph adds four new vertices and multiplies by 24 the total number of possible embeddings.

To get a better bound we use the following observation.

Lemma 5.5. A small perturbation of the lengths of the moving edges of the 4-bar mechanism from Lemma 5.3 yields slightly perturbed coupler curves, which still intersect the two symmetric circles in 24 points.

We now fix a bar in the plane, place on it a 4-bar mechanism satisfying Lemma 5.3, fix the positions of the symmetric triangles and the corresponding circles that give the 24 embeddings. Then perturb the 4-bar mechanism (but not the base triangle) several times as in Lemma 5.5, to obtain a fan-like gluing of Desargues configurations. See Fig. 6(b). Then the same two symmetric circles (or circles of slightly perturbed radii, but with the same center) cross each pair of coupler curves (one pair for each of these mechanisms) in 24 points, giving the desired number of embeddings.

Proposition 5.6. There exist edge lengths for which the number of embeddings of the iterated "fan" Desargues frameworks is of the order of $2 \times 12^{n / 3} \approx(2.28)^{n}$.

Proof. Each Desargues graph now adds three new vertices and 12 possible embeddings, since we keep the same base triangle. This results in $12^{n / 3}$ embeddings, doubled by symmetry with respect to the pinned down base edge.

As a final comment, we note that we can combine the perturbation idea from the previous construction with the "caterpillar structure" by gluing triangle over triangle (instead of bar over bar) to get another family of examples achieving the same bound.

\section{Upper Bounds in the Non-Euclidean Case}

Since one may consider the analogous problem in non-Euclidean spaces (of constant sectional curvature), we outline here an adaptation of our Euclidean approach which yields upper bounds for the spherical and hyperbolic situations.

We denote by $S^{d}$ the unit sphere in $R^{d+1}$ and describe the argument for this case. The hyperbolic case $H^{d}$ will then obey the same bound "by analytic continuation" (from 1 to $i$ ), since all relevant equations involve cosines in spherical geometry, respectively hyperbolic cosines in hyperbolic geometry, and

$$
\cosh (\theta)=\cos (i \theta) .
$$

For realizations $G(p)$ in $S^{d}$ of graphs with prescribed edge lengths $l_{i j}$, one requires $0<l_{i j}<\pi$ so that the edge from $p_{i}$ to $p_{j}$ always runs along the shorter arc of the grand circle passing through $p_{i}$ and $p_{j}$.

A first remark is that the class of $d$-minimally rigid graphs is the same irrespective of context (spherical, Euclidean or hyperbolic), that is, a graph which is $d$-minimally rigid in one sense will be $d$-minimally rigid in the other two as well. 
Now, the $d$-dimensional spherical realizations of a $d$-minimally rigid graph $G$ correspond precisely with the $(d+1)$-dimensional Euclidean realizations of the graph $\tilde{G}$ with $n+1$ vertices, obtained from $G$ by introducing a new vertex $v_{0}$ connected to all previous vertices by edges of prescribed length 1 , while the remaining edges are adapted in length from arcs to chords. (Obviously, the new vertex will be the center of $S^{d}$ in all realizations.)

The role played by the complex Cayley-Menger variety $C M^{d, n}(C)$ for $n$ points (vertices) in $R^{d}$ will be taken by $C M^{d+1, n+1}(C)$ intersected with $n-1$ hyperplanes $d_{01}=d_{02}=\cdots=d_{0 n}$, for $n$ points (vertices) in $S^{d}$.

Since intersections with hyperplanes do not change degrees, we obtain Theorem 1.4 in the Introduction:

Theorem 6.1. Let $d$ be given and assume a generic choice of edge lengths (subject to $0<l_{i j}<\pi$ in the spherical case). Ad-minimally rigid graph with $n \geq d+1$ vertices has at most

$$
2 D^{d+1, n+1}=2 \prod_{k=0}^{n-d-2} \frac{\left(\begin{array}{c}
n+k \\
n-d-1-k
\end{array}\right)}{\left(\begin{array}{c}
2 k+1 \\
k
\end{array}\right)}
$$

realizations in the sphere $S^{d}$, or the hyperbolic space $H^{d}$, up to orientation preserving isometries (i.e. rigid motions).

\section{Acknowledgements}

We thank Brigitte Servatius and Walter Whiteley for pointing out useful references.

\section{References}

1. S. Basu, Different bounds on the different Betti numbers of semi-algebraic sets, Proc. 17th Symp. on Comput. Geometry (SoCG), Medford, MA, 2001, pp. 288-292.

2. S. Basu, R. Pollack and M.-F. Roy, Algorithms in Real Algebraic Geometry, in preparation.

3. A. Berg and T. Jordán, A proof of Connelly's conjecture on 3-connected circuits of the rigidity matroid, J. Combin. Theory Ser. B, to appear.

4. M. Berger, Geometry, Vols. I and II, Springer-Verlag, New York, 1987.

5. L. Blumenthal, Theory and Applications of Distance Geometry, Chelsea, New York, 1970.

6. C. Borcea. Point configurations and Cayley-Menger varieties, manuscript, http://arxiv. org/abs/ math/0207110, 2002.

7. J. Canny, Complexity of Robot Motion Planning, MIT Press, Cambridge, MA, 1988.

8. A. L. Cauchy, Sur les polygones et les polyhèdres, XVIe Cahier IX, 1813, pp. 87-89.

9. R. Connelly, Rigidity, in Handbook of Convex Geometry, vol. A (P. M. Grüber and J. M. Wills, eds.), North Holland, Amsterdam, 1993, pp. 223-272.

10. G. M. Crippen, Distance Geometry and Conformational Calculations, Research Studies Press, Wiley, New York, 1981.

11. G. M. Crippen and T. F. Havel, Distance Geometry and Molecular Conformation, Research Studies Press, Wiley, New York, 1988.

12. M. Dehn, Über die Starrheit konvexer Polyeder, Math. Ann. 77 (1916), 466-473.

13. M. M. Deza and M. Laurent, Geometry of Cuts and Metrics, Springer-Verlag, New York, 1997.

14. W. Fulton, Intersection Theory, Springer-Verlag, New York, 1984. 
15. G. Z. Giambelli, Sulle varietá rappresentate coll'annullare determinanti minori contenuti in un determinante simmetrico od emisimmetrico generico di forme. Atti R. Accad. Sci. Torino 44 (1905/1906), $102-125$.

16. H. Gluck, Almost all simply connected closed surfaces are rigid, in Geometric Topology, Lecture Notes in Mathematics, No. 438, Springer-Verlag, Berlin, 1975, pp. 225-239.

17. J. Graver, B. Servatius and H. Servatius, Combinatorial Rigidity, Graduate Studies in Mathematics, vol. 2, American Mathematical Society, Providence, RI, 1993.

18. J. Harris, Algebraic Geometry, Graduate Texts in Mathematics 133, Springer-Verlag, New York, 1993.

19. J. Harris and L. W. Tu, On symmetric and skew-symmetric determinantal varieties, Topology 23 (1984), 71-84.

20. B. Hendrickson, The Molecule Problem: Determining Conformation from Pairwise Distances, Technical Report 90-1159, Ph.D. Thesis, Computer Science Department, Cornell University, 1990.

21. B. Hendrickson, Conditions for unique graph realizations, SIAM J. Comput. 21(1) (1992), 65-84.

22. L. Henneberg, Die graphische Statik der starren Systeme, Leipzig, 1911.

23. J. A. Hrones and G. I. Nelson, Analysis of the Fourbar Linkage, MIT Technology Press, Cambridge, MA, 1951.

24. T. Józefiak, A. Lascoux and P. Pragacz, Classes of determinantal varieties associated with symmetric and skew-symmetric matrices, Math. USSR-Izv. 18 (1982), 575-586.

25. G. Laman, On graphs and rigidity of plane skeletal structures, Eng. Math. 4 (1970), 331-340

26. J. Milnor, On the Betti numbers of real varieties, Proc. Amer. Math. Soc. 15 (1964), 275-280.

27. R. L. Norton, Design of Machinery, 2nd edn., McGraw-Hill, New York, 2001.

28. O. A. Oleinik and I. B. Petrovskii, On the topology of real algebraic surfaces, Izv. Akad. Nauk SSSR 13 (1949), 389-402.

29. J. Richter-Gebert and U. Kortenkamp, Cinderella, an Interactive Software Package for Geometry, Springer-Verlag, New York, 1999.

30. J. B. Saxe, Embedability of weighted graphs in $k$-space is strongly NP-hard, Proc. Allerton Conf. on Communications, Control and Computing, 1979, pp. 480-489.

31. E. Steinitz and H. Rademacher, Vorlesungen über die Theorie der Polyedern, Springer-Verlag, Berlin, 1934.

32. T.-S. Tay and W. Whiteley, Generating isostatic graphs, Structural Topology 11 (1985), 21-68.

33. R. Thom, Sur l'homologie des variétés réelles, Differential and Combinatorial Topology, Princeton University Press, Princeton, NJ, 1965, pp. 255-265.

34. W. Whiteley, Matroids from Discrete Applied Geometry, Contemporary Mathematics, vol. 197, American Mathematical Society, Providence, RI, 1996, pp. 171-311.

35. W. Whiteley, Rigidity and scene analysis, in Handbook of Discrete and Computational Geometry (Jacob E. Goodman and Joseph O’Rourke, eds.), 1997, pp. 893-916.

36. W. Whiteley, Rigidity of molecular structures, in Rigidity Theory and Applications (Thorpe and Duxbury, eds.), Kluwer, Dordrecht, 1999.

37. W. Wunderlich, Hohere Koppelkurven, Österreich. Ingen. Arch. XVII(3) (1963), 162-165.

Received July 15, 2002, and in revised form December 18, 2002. Online publication December 31, 2003. 